\title{
GENERALIZED SUM-FREE SUBSETS
}

\author{
YAIR CARO \\ School of Education \\ University of Haifa - Oranim \\ Tivon - 36910 \\ Israe 1
}

(Received November 21, 1989)

ABSTRACT. Let $F=\{A(i): 1<1<t, t>2\}$, be a finite collection of finite, pairwise disjoint subsets of $z^{+}$. Let $S \subset R \backslash\{0\}$ and $A \subset z^{+}$be finite sets. Denote by $S^{A}=\left\{\sum_{1}^{a} s_{1}: a \in A, S_{1} \varepsilon S\right.$, the $s_{1}$ are not ncesarily distinct $\}$. For $S$ and $F$ as above we say that $S$ is $F$-free if for every $A(1), A(j) \in F, i \neq j, s^{A(1)} \cap s^{A(j)}=\phi$.

We prove that for $S$ and $F$ as above, $S$ contalns an $F-f$ ree subset $Q$ such that $|Q|>c(F)|S|$, when $c(F)$ is a positive constant depending only on $F$.

This result generalizes earlier results of Erdos [3] and Alon and Kleitman [2], on sum-free subsets. Several possible extensions are also discussed.

\section{INTRODUCTION.}

A set $S$ of integers is called sum-free if $(S+S) \cap S=\emptyset$, 1.e. if there are not (not necessarily distinct), $a, b, c \in S$ such that: $a+b=c$. There is a considerable amount of results concerning sum-free subsets, not only within the integers, but also in the context of abelian groups. The monumental survey by Wallis and Street [1] is recommended for that purpose. Recent1y Alon and Kleitman [2] proved (among many other interesting results) the following theorem.

THEOREM A:[2]. Any finite set B of nonzero reals contains a sum-free subset $A$ of cardinality $|A|>|B| / 3$.

This 1s a s1ight 1mprovement of an old result of Erdos [3]. Here we consider the wore general problem mentioned in the abstract. Let us first recall the exact formulation of the generalized sum-free subset problem. Let $F=\{A(1): 1<1<t, t>2\}$, be a finite collectilon of finite, palrwise disjoint, subsets of $\mathrm{z}^{+}$. Let $A \subset \mathrm{z}^{+}$and $S \subset R \backslash\{0\}$ be finite sets. Denote by $S^{A}=\left\{_{1}{\stackrel{q}{1} s_{1}}_{1}\right.$ a $\varepsilon A, s_{1} \in S$, the $s_{1}$ are not necessarily distinct $\}$. For $S$ and $F$ as defined above we say that $S$ is $F$-free if for every $A(1), A(j) \in F, 1 \neq j$, $s^{A(1)} \cap s^{A(1)}=0$. Clearly for $F=\{\{1\},\{2\}\}$ this is fust the case of sum-free subsets investigated in [2] and [3].

Erdos as well as Alon and Kleftman gave a probabilistic proof of Theorem A. We shall give a proof of Theorem $A$ in the case of integers, avoiding the probabilistic tools and using a double-counting instead. Our maln goal however is to generalize 
both the content and the proof technique of Theorem $A$ to an arbitrary collection $F$ as explained above. We shall combine the probabilistic ideas of [2] together with an observation on non-intersecting subintervals of the unit interval $[0,1)$. We prove the following:

THEOREM 1.1. Let $S$ be a finite subset of $R \backslash\{0\}$. Let $F=\{A(i): 1<i<t, t>2\}$ be a finite scollection of finite, pairwise disjoint, subsets of $z^{+}$, then $S$ contains an F-free subset $Q$ of cardinality $|Q|>c(F)|S|$, when $c(F)$ is a positive constant depending on $\mathrm{F}$ on $1 \mathrm{y}$.

2. PROOFS OF THE THEOREMS.

We first give a non-probabilistic proof of Theorem $A$ in the case of integers.

PROOF OF THEOREM $A$. Let $B=\left\{b_{1}, b_{2}, \ldots, b_{n}\right\}$ be a set of integers. Let $p=2 k+2$ be a prime number such that: $p>2 \max _{i}\left|b_{i}\right|$, and put $c=\{k+1, k+2, \ldots, 2 k+1\}$. observe that $C$ is a sum-free subset of the cyclic group $Z_{p}$, and that $|c| /(p-1)=$ $(k+1) /(3 k+1)>1 / 3$.

For any $x, 1<x<p-1$, define $d_{i}(x)=d_{i}$ by $d_{i} \equiv x b_{1}(\bmod p), \quad 0<d_{i}<p$. Clearly, for every fixed $i, 1<i<n$, as $x$ ranges over all numbers $1,2 \ldots, p-1, d_{1}$ ranges over all nonzero elements of $z_{p}$.

Now we use double-counting instead of the probablistic argument. For every $b_{i}, 1<i<n$, let $t\left(b_{i}\right)=\left|\left\{x: d_{i}=x b_{i} \varepsilon c\right\}\right|$. For every $x, 1<x<p$, let $r(x)=\left|\left\{i: d_{1}=x b_{i} \varepsilon c\right\}\right|$. By double-counting we have $\sum_{i=1}^{n} t\left(b_{i}\right)=\sum_{x} r(x)$, but by the cholce of $P, t\left(b_{1}\right)=k+1$ for $1<1<n$. Hence for some $1<x<p-1$, $r(x)>n(k+1) /(p-1)=n(k+1) /(3 k+1)>n / 3$. Consequently there is a subset $A$ of $B$, of cardinality $|A|>|B| / 3$ such that $x a(\bmod p) \varepsilon C$, for all a $\varepsilon A$. This subset A is sum-free, since if $a+b=c$, for some, $a, b, c \varepsilon A$ then $x a+x b=x c(\bmod p)$, which is impossible because $C$ is sum-free in $Z_{p}$. Q.E.D.

The proof of Theorem A rests on the basic idea which is to find a "large" sumfree subinterval of $Z_{p}$ and to map the set, under consideration, onto $z_{p}$ such that a large portion of $i t$, is mapped onto the sum-free subinterval. We shall apply such an idea in the following proof of Theorem 1.

PROOF OF THEOREM 1. Let $A(i) \in F$ be a finite set of positive integers and order them in an ascending order, such that $A(i)=\left\{a(1,1)<a(i, 2)<\ldots<a\left(1, n_{i}\right)\right\}$. Denote by $\delta=\delta(F)=\min \{a(1,1): 1<1<n\}$. Consider the sequence $B(F)$ of the largest elements of each set $A_{i}$, namely $B(F)=\left\{a\left(i, n_{1}\right): 1<i<n\right\}$. Denote by $L_{1}(F)$ resp. $\mathrm{L}_{2}(\mathrm{~F})$ the largest resp. the second largest element of $B(F)$. Finally let $r=r(F)=$ $\max \left\{x \in R: L_{2}(F)<x<L_{1}(F), \quad x / L_{2}(F)<\min \{a(k, 1) / a(i, j): i \neq k, a(k, 1)>a(i, j)\}\right\}$.

We are going to show that $c(F)$, the constant in Theorem 1 , is at least

$c(F)>\left(r-L_{2}(F)\right) /\left(r L_{1}(F)-\delta L_{2}(F)\right)>0$. First we show that the interval

$[\alpha, \beta) \subset[0,1]$ is $F-f$ ree with respect to addition modulo-1, with the choice $: \alpha=\mathrm{L}_{2}(\mathrm{~F}) /\left(\mathrm{rL}_{1}(\mathrm{~F})-\delta \mathrm{L}_{2}(\mathrm{~F})\right)$, and $\beta=\mathrm{r} /\left(\mathrm{rL}_{1}(\mathrm{~F})-\delta \mathrm{L}_{2}(\mathrm{~F})\right)$. From the definitions $r L_{1}(F)-\delta L_{2}(F)>0$, hence $0<\alpha<\beta$. Moreover, since $\delta L_{2}(F)<r\left(L_{1}(F)-1\right)$, we infer that $\beta<1$, hence it follows that $[\alpha, \beta) \subset[0,1]$. Now suppose there are 
$a(1, j) \in A(i)$ and $a(k, 1) \in A(k)$, such that $a(k, 1)>a(i, j)$ and $a(k, 1)[\alpha, \beta)(\bmod 1) \cap a(1, j)[\alpha, \beta)(\bmod 1)=0 .(c[\alpha, \beta)=:(c \alpha, c \beta))$. Observe that because of the specific cholce of the parameters $\alpha$, $\beta$ and since $\beta L_{1}(F)=1+\delta \alpha$ as can be seen by a direct verification, we actually must have an intersection even without (mod 1) consideration, namely we must have $a(k, 1)[\alpha, \beta) \cap a(i, j)(\alpha, \beta)=0$. Hence it follows that $a(i, j) B>a(k, l) \alpha$, which $\ln$ turn implies that $r / L_{2}(F)=\beta / \alpha>$ $a(k, 1) / a(i, j)>1$, a contradiction to the choice of $r=r(F)$. Hence with respect to addition modulo-1, $(\alpha, \beta)$ is indeed $F-f$ ree. Now let $\mu=\min \left|s_{i}\right|$. We choose, randomly, a real number $x$ according to a uniform distribution on the interval $I$, defined by $I=[1 / \mu, n \theta(F) / \mu]$, when $\theta(F)$ is a large constant depends only on $F$, and compute the numbers $d_{i}(x)=: d_{1}=\left(x s_{1}\right)$ (mod 1$)$. Observe that for fixed $1,1<1<n$, every subinterval of $I$ of length $1\left|s_{1}\right|$ is mapped onto $[0,1)$, hence for large $\theta(F)$ the expected number of $d_{1}-8$ that belongs to $[\alpha, \beta)$ is more than $(\beta-\alpha)(n-1)$ and hence there is an $x$ and a subset $Q$ of at least $(\beta-\alpha)$ n members of $S$ such that (xq) $(\bmod 1) \varepsilon(\alpha, \beta)$ for each $q \varepsilon Q$. But now we are done, because $(\alpha, \beta)$ is F-free with respect to addition modulo 1 and also $\beta-\alpha=\left(r-L_{2}(F)\right) /\left(r L_{1}(F)-\delta L_{2}(F)\right)$ as needed.

As a demonstration of the parameter involved let $A(1)=\{1,4,7\}, A(2)=\{2,5,8\}$, $A(3)=\{3,6,9\}$ and $F=\{A(1), A(2), A(3))$. Then $\delta(F)=1, B(F)=7,8,9\}$, $L_{1}(F)=9, L_{2}(F)=8, r(F)=9$ and $c(F)=(9-8) /(9 * 9-1 * 8)=1 / 73$. Moreover the interval $[8 / 73,9 / 73)$ is $F-f$ ree with respect to addition modulo-1.

\section{EXTENSIONS AND VARIATIONS.}

1. A set of linear equations over $Z$.

A natural question that can be asked now 1s, what can we say, for example, about the following set of linear equations:

$$
\begin{gathered}
5 a+3 b+2 c=9 x \\
3 a+3 b+3 c+d=8 x+y \\
4 a+2 b+2 c+2 d=6 x+2 y+z
\end{gathered}
$$

One can easily check that these equations are special cases of the F-free problem. Indeed we on1y have to define $A(1)=\{10\}, A(2)=\{9\}$, and $F=\{A(1), A(2)\}$. Any

F-free subset contains no solution of any of the above equations. This observation can be set in a general form as follows.

THEOREM 3.1. Let $\sum_{1=1}^{j} a(1, j) x_{1}=\sum_{1=1}^{j} b(1, j) y_{1}, 1<j<n$, be a set of $n$ linear equations with positive integer coefficients. Suppose further that for any $u$ and $v, \sum_{1=1}^{\sum_{1}} a(1, u) \neq \sum_{i=1}^{v} b(1, v)$, then any set $S$ of nonzero reals contains a subset $Q$, of cardinality $|Q|>c|s|$, when $c$ is a positive constant depending only on the sums of the coefficients of the equations, and such that no linear equation of the prescribed set is solvable within $Q$.

PROOF. Observe that we only have to define $A(1)=\left\{\sum_{1} \sum_{j}^{n} a(1, j), \quad 1<j<n\right\}$, $A(2)=\left\{1 \sum_{1}^{j} b(1, j), 1<j<n\right\}$, and $F=\{A(1), A(2)\}$. By Theorem 1 we are done, because any $F-f$ ree subset of $S$ contains no solution of any of the equations.

2. A set of linear equations over $Q$.

Lets' have a look at the following linear equations over Q: 


$$
\begin{aligned}
& 5 a / 3+2 b / 9=2 x / 7+5 y / 14 \\
& a / 3+b / 3+c / 3=x / 2+y / 2
\end{aligned}
$$

There is an essential difference between them. The first one can be transformed into the equation

$$
210 a+28 b=36 x+45 y \text {. }
$$

Clearly $210+28=238 \neq 81=36+45$. Hence Theorem 3.1 can be applied here with $A_{1}=\{238\}$, and $A_{2}=\{81\}$. The second equation can be transformed into $2 a+2 b$ $+2 c=3 x+3 y$, but now $2+2+2=6=3+3$, and we can't use Theorem 1 or Theorem 3.1 , because the condition $A(1) \cap A(2)=\emptyset$ is violated. However from the examples given above it is clear that Theorem 3.1 remains valid in the more general situation of positive rational coefficients, and that a generalization of Theorem 1 to the case of rationals is possible. We omit the quite obvious details.

3. There is still the question of what can be said, if anything, in the case when for some $A(i), A(j) \varepsilon F, A(i) \cap A(j) \neq \emptyset, e \cdot g a+b=2 c$ or $a+2 b+3 c+4 d=10$ e etc. We hope to comment about such problems in Wallis, Street, and Wallis [4] under the frame of independent sets of hypergraphs. One can see that even the simplest case $a \div b=2 c$, is closely related to the we11 known Theorem of Szemeredi on arthmetic progressions. So it is unreasonable to expect that results like Theorem 1 and Theorem 3.1 can hold in this case.

\section{TWO OPEN PROBLEMS.}

1. The most interesting open problem is that of determining the best possible constant in Theorem 1 , with respect to a given set $F$, and in particular for the case of sum-free sets to prove or disprove that $1 / 3$ is the best possible constant.

2. Another interesting problem is to obtain a constant better than $1 / 3$ in the case when $S$ is a set of squares of nonzero integers. Here we hve to avoid $a^{2}+b^{2}=$ $c^{2}$. Clearly we can't hope for a constant better than $2 / 3$ because we can take a large set consisting of arbitrarily large pythagorian's triples.

ACKNOWLEDGEMENT. I would like to thank Noga Alon for his help and inspiration.

\section{REFERENCES}

1. WALliS, W.D., STREET, A.P. and WALlis, J.S. Combinatorics, Room Squares, Sumfree Sets, Hadamard Matrices, Lecture Notes in Mathematics 292, Springer Verlag, Ber11n, Heidelberg and New York, (1972), Part 3, 123-277.

2. ALON, N. and KLEITMAN, D.J. Sum-free subsets, Preprint to appear 1989.

3. ERDOS, P. Extremal problems in number theory, Proc. Symp. Pure Math. 8 AMS (1965), 181-189.

4. CARO, Y. and TUZA, Zs. Indpendent sets in hypergraphs, in preparation. 


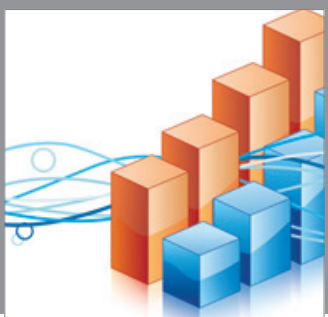

Advances in

Operations Research

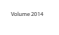

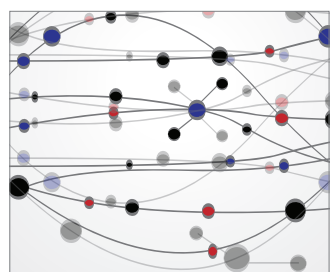

\section{The Scientific} World Journal
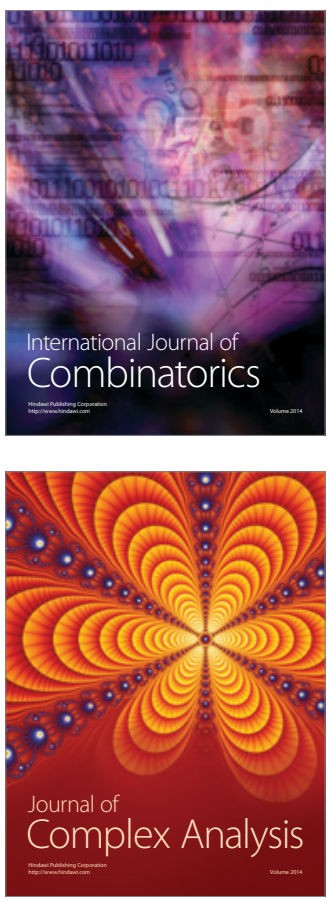

International Journal of

Mathematics and

Mathematical

Sciences
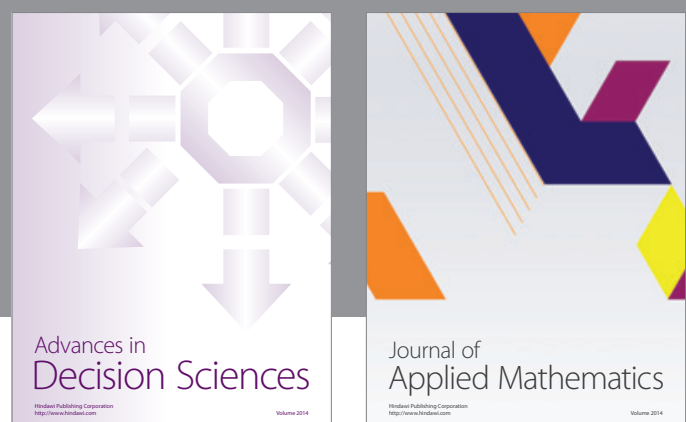

Journal of

Applied Mathematics
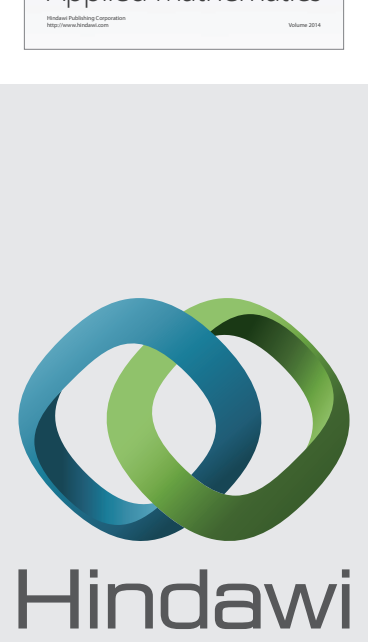

Submit your manuscripts at http://www.hindawi.com
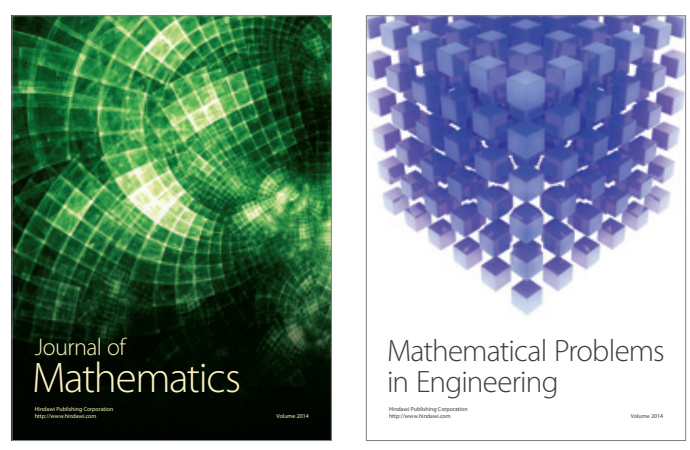

Mathematical Problems in Engineering
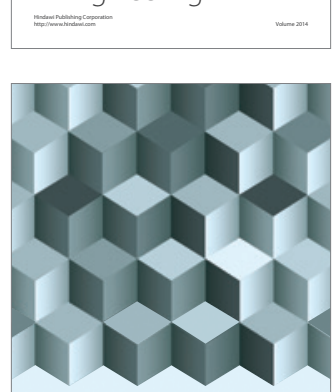

Journal of

Function Spaces
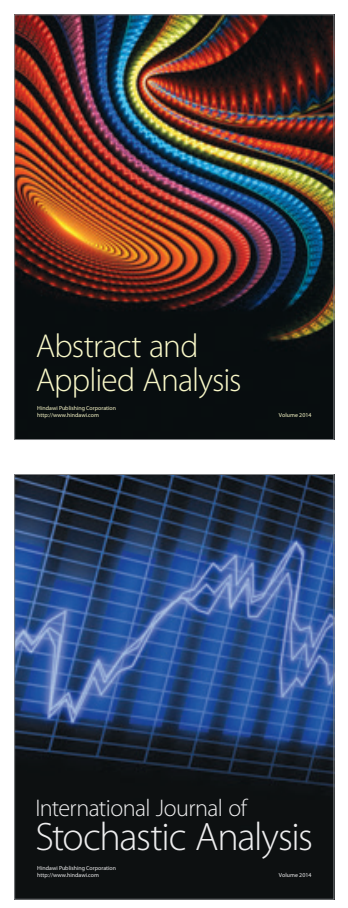

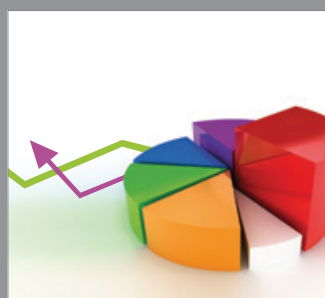

ournal of

Probability and Statistics

Promensencen
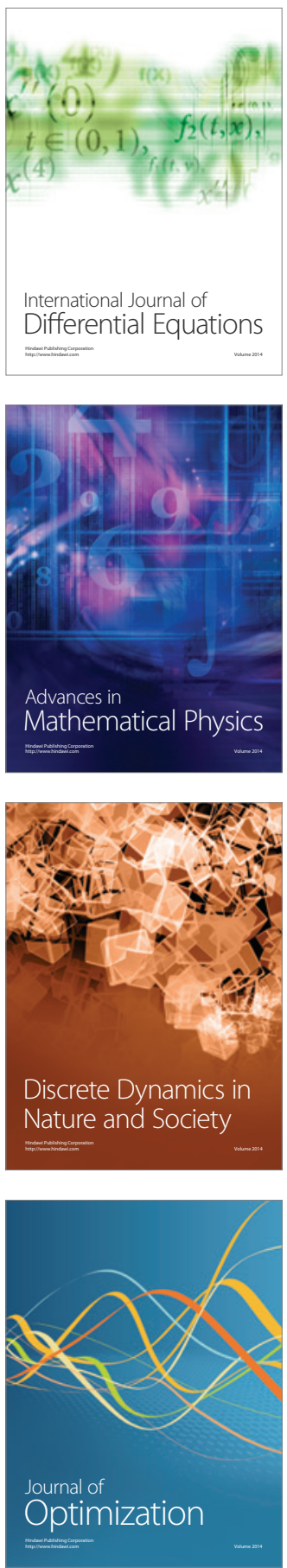